\title{
The Development of Local, Layer-Specific Visual Cortical Axons in the Absence of Extrinsic Influences and Intrinsic Activity
}

\author{
Jami L. Dantzker and Edward M. Callaway \\ Systems Neurobiology Laboratories, The Salk Institute for Biological Studies, La Jolla, California, 92037, and Department \\ of Biology, University of California San Diego, La Jolla, California 92093
}

\begin{abstract}
The laminar specificity of vertical connections in the primary visual cortex (area 17) develops precisely from the outset, leading to the hypothesis that layer-specific axonal targeting is attributable to molecular cues intrinsic to the cortex (Lund et al., 1977; Katz and Callaway, 1992). However, alternative factors that could influence axonal development have not been investigated. This study examines the roles of intrinsic cortical activity and extrinsic influences that could arise from earlierformed connections with outside cortical and subcortical areas. Organotypic slice cultures were prepared from ferret area 17 before the formation of local axonal connections and were incubated for 5-7 d to allow initial, local axonal arbors to form in the absence of extrinsic influences. Additionally, some slices were cultured in the presence of the $\mathrm{Na}^{+}$channel blocker tetrodotoxin to block spontaneous action potentials within the
\end{abstract}

slice. Individual neurons were labeled intracellularly with biocytin, and their patterns of local axonal arborizations were reconstructed. This study focuses on the development of layer 6 pyramidal neurons, the axons of which in vivo bypass an incorrect target, layer 5 , before specifically arborizing in their local target, layer 4 . We found that axonal arbors developing in vitro preferentially arborized in layer 4 versus layer 5 . However, inhibition of spontaneous activity within the cortical slice decreased this specificity, resulting in similar numbers of axonal branches in layers 4 and 5. Thus, although cortical axons do not require influences from outside areas, intrinsic spontaneous activity is required for specific axonal arborization in correct laminar targets.

Key words: ferret; local circuits; area 17; organotypic slice culture; intracellular label; tetrodotoxin; spontaneous activity
The mammalian cerebral cortex is composed of precisely wired connections critical to cortical function. A common organizational feature is a specific network of axonal connections between the four main cortical layers (2/3, 4, 5, and 6) (Gilbert, 1983; Martin and Whitteridge, 1984; Lund, 1988). These local, interlaminar connections develop precisely from the outset. Neurons do not make initial connections in nontarget layers that are later pruned back to produce the specific adult pattern (Lund et al., 1977; Katz, 1991; Callaway and Katz, 1992; Callaway and Lieber, 1996; Callaway 1998).

These observations of precise initial formation of layer-specific connections have led to the hypothesis that cortical axons respond to activity-independent cues intrinsic to the cortical layers, such as extracellular molecular markers (Katz and Callaway, 1992; Goodman and Shatz, 1993). In support of this idea, it was shown recently that axons from rat ventricular zone explants containing mostly layer 6 cells $(\sim 80 \%)$ grew and branched preferentially on membrane carpets prepared from target layers of postnatal cortex, as opposed to those prepared from nontarget layers (Castellani and Bolz, 1997). The above in vitro experiments examined the combined axonal arbor patterns for large, heterogeneous populations of neurons that grew axons premature to their normal

\footnotetext{
Received Jan. 8, 1998; revised March 3, 1998; accepted March 9, 1998.

This work was supported by National Institutes of Health Grant EY10742 and National Science Foundation Graduate Research Fellowship (J.L.D.). We thank Dr. C. F. Stevens for providing culture facilities, Dr. H. Kida, Dr. A. Herzog, K. Reese, and A. Sawatari for technical assistance, M. S. Dantzker, D. Irwin, and Dr. T. Price for helpful discussions on analyses, and Dr. A. K. McAllister for helpful comments on this manuscript.

Correspondence should be addressed to Jami Dantzker, Systems Neurobiology Laboratories-C, The Salk Institute, 10010 North Torrey Pines Road, La Jolla, CA 92037.

Copyright (ㄷ) 1998 Society for Neuroscience $\quad 0270-6474 / 98 / 184145-10 \$ 05.00 / 0$
}

developmental time period. Although the results support a potential role for intrinsic molecular cues, this approach does not address subtle effects on the degree of axonal specificity that might be elicited by changes in cortical environment during initial arbor development.

We are interested in determining whether alternative mechanisms play a role in the formation of local, layer-specific connections for individual pyramidal neurons during the time they would first begin to grow into their target layers. Neurons in area 17 are reciprocally connected with extrinsic targets before and during development of local connections [e.g., lateral geniculate nucleus (LGN) and superior colliculus; O'Leary and Koester, 1993]. Such extrinsic areas might influence the cortical environment in several ways. For example, afferents could influence local signaling pathways and/or express molecular cues on their axons (Mozer and Benzer, 1994; Habecker et al., 1995; Fryer et al., 1996; Kawamoto et al., 1996). Extrinsic target areas could also retrogradely influence molecular expression through cortical efferents (Stevens and Landis, 1988; Williams et al., 1994; Wu et al., 1997).

Afferents could also influence the cortex in an activitydependent manner. For example, LGN axons that arborize in layers 6 and 4 could influence the activity-dependent secretion of trophic molecules (Thoenen, 1995; Bonhoeffer, 1996).

Likewise, spontaneous cortical activity could play a role in the formation of layer-specific connections by affecting the expression of molecular cues or receptors for cue detection (Blochl and Thoenen, 1995; Goodman et al., 1996; Xie et al., 1997) or by selectively reinforcing connections (Changeux and Danchin, 1976). Although retinal activity may not be required for normal development of layer-specific connections as suggested by binocular deprivation experiments (Callaway and Katz, 1992), this 
P13-15

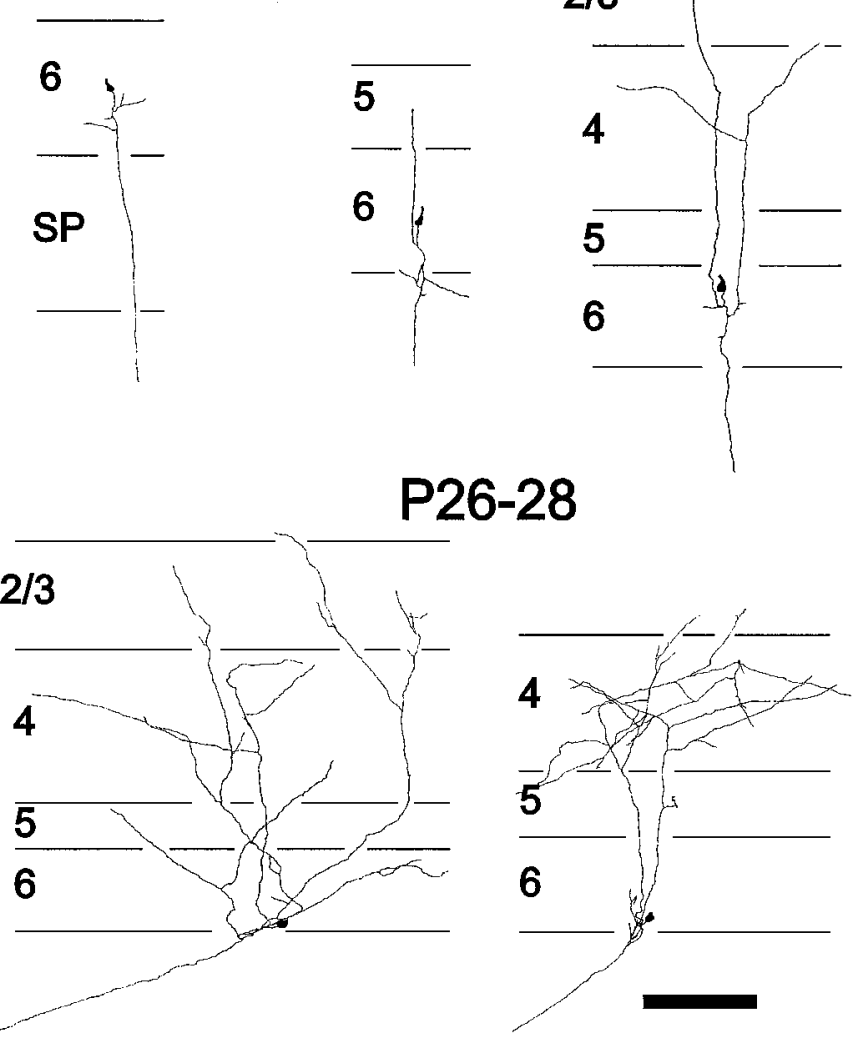

Figure 1. Summary of in vivo development of axonal arbors from ferret layer 6 pyramidal neurons. Dendrites are omitted for clarity. At P13-15 layer 6 pyramids have not yet sent an axon collateral into the overlying cortical layers but have a single descending axon extending into the white matter. By P19-20, neurons have a few recurrent axon collaterals that are just beginning to form branches in layer 4 . Later in development, the density of axonal arbors continues to increase in layers 4 and 2/3, with very few arbors ever forming in layer 5. Figure modified from Callaway and Lieber (1996). Scale bar, $200 \mu \mathrm{m}$.

does not rule out all activity-dependent mechanisms. Binocular deprivation does not block spontaneous activity, and even binocular enucleation does not prevent the development of clustered horizontal connections that is dependent on cortical activity (Ruthazer and Stryker, 1996).

To address possible alternative mechanisms, we made organotypic slice cultures of area 17 from young ferrets before the growth of axonal projections from layer 6 pyramidal neurons to more superficial cortical layers (Fig. 1) (Callaway and Lieber, 1996). Layer 6 axons in culture exhibited an arborization pattern very similar to that seen in vivo; they bypassed their incorrect target, layer 5, and arborized specifically in layer 4 and partially in layer $2 / 3$. However, blocking spontaneous activity in cortical slices with the $\mathrm{Na}^{+}$channel blocker tetrodotoxin (TTX) decreased this specificity. Therefore, intrinsic molecular cues appear to instruct the formation of initial layer-specific connections within the cortex, but spontaneous activity may influence the detection or expression of these cues.

\section{MATERIALS AND METHODS}

Preparation and culturing of cortical slices. Area 17 was removed and sliced from nine ferrets, ages postnatal days 14-15 (P14-P15), as described previously (Callaway and Lieber, 1996) but under sterile condi- tions. Animals were deeply anesthetized with sodium pentobarbital (100 $\mathrm{mg} / \mathrm{kg}$, i.p.) and decapitated, and the brains were removed and placed in ice-cold HEPES-buffered artificial CSF (aCSF; in mM: $140 \mathrm{NaCl}, 5 \mathrm{KCl}$, $1 \mathrm{MgCl}_{2}, 1 \mathrm{CaCl}_{2}, 24$ D-glucose, and 10 HEPES, pH 7.4). Area 17 was dissected from the cortical tissue and sagittally sliced at $400 \mu \mathrm{m}$ using an "egg-slicer"-like device (Katz, 1987). After at least a 30 min incubation in ice-cold HEPES-buffered aCSF, slices were then transferred onto the membrane of a tissue culture insert $(0.4 \mu \mathrm{m}$ pore size, Falcon; Franklin Lakes, NJ) and placed in 6-well culture dishes with culture media $(50 \%$ basal medium eagle (BME) with glutamine, 25\% HBSS, $25 \%$ horse serum, $6.5 \mathrm{mg} / \mathrm{ml}$ dextrose, and $10 \mathrm{mM}$ HEPES) that formed an airliquid interface. To inhibit $\mathrm{Na}^{+}$-dependent action potentials, $1 \mu \mathrm{M}$ TTX was added to the media of half of the cultures from the start of incubation. All cultures were then covered and placed in a tissue culture incubator at $37^{\circ} \mathrm{C}$ and $5 \% \mathrm{CO}_{2}$. Media were replaced every 2-3 d, including TTX in the appropriate cultures. Less than half of the total cultures received low-serum media (10\% horse serum, $65 \%$ BME with glutamine, $25 \%$ HBSS, $6.5 \mathrm{mg} / \mathrm{ml}$ dextrose, $10 \mathrm{~mm}$ HEPES, $2 \% \mathrm{~N}-2$ supplement, $35 \mathrm{~nm} 5$-Fluoro-2'-Deoxyuridine antimitotic agent, $75 \mathrm{~nm}$ uridine, and $5 \mathrm{ng} / \mathrm{ml}$ penicillin-streptomycin) 2-3 d before removal for intracellular labeling. This change did not differently affect the growth of axons.

Slices were incubated for 5-7 d in culture (dic), which corresponds to P19-P22 in vivo, and then removed for intracellular labeling with biocytin. Membranes below the slice were cut away from the rest of the culture insert, and the slice was placed in an oxygenated interface holding chamber with carbonate-buffered aCSF (Callaway and Lieber, 1996). Slices had reduced in thickness to $\sim 100-200 \mu \mathrm{m}$ in culture.

Intracellular labeling and tissue processing. Slices were labeled intracellularly as described previously (Callaway and Lieber, 1996; Callaway and Wiser, 1996). In short, after incubating in an oxygenated interface chamber for $\sim 1 \mathrm{hr}$, the slices were submerged in a recording chamber with oxygenated and warmed aCSF. Whole-cell recordings were made in current-clamp mode using patch electrodes (Blanton et al., 1989). Two percent biocytin (Sigma, St. Louis, MO) was included in the intracellular solution and iontophoresed into the cell using positive current. To verify the effectiveness of TTX in blocking $\mathrm{Na}^{+}$channels, we injected $0.3-0.5$ $\mathrm{nA}$ of step current into cells immediately after obtaining recordings $\sim 5-15$ min after slice removal from TTX-treated media. This was done for one slice from each culture well (there were usually four slices per well). No action potentials were detected in TTX-treated neurons within this time, but an action potential usually slowly emerged, because the TTX began to wash out. Action potentials were always detected in neurons never exposed to TTX. There was no difference in the ability to patch onto cells or apparent health of the slices between the TTX- and non-TTX-treated slices.

After postlabeling incubation, slices were fixed in $4 \%$ paraformaldehyde, and labeled cells were stained using a horseradish peroxidaseconjugated avidin-biotin complex (Peroxidase Standard Kit; Vector Laboratories, Burlingame, CA). Slices were mounted on gelatin-coated slides, stained for Nissl substance with thionin to allow detection of laminar boundaries (Callaway and Lieber, 1996), dehydrated, and coverslipped with Permount.

Analyses of labeled neurons. Neuronal processes were reconstructed using camera lucida light microscopy and a $40 \times[1.0$ numerical aperture $(\mathrm{NA})]$ or $63 \times(1.4 \mathrm{NA})$ oil immersion objective. Only neurons with completely labeled axons and dendrites were used in our analyses; no layer 6 pyramidal neurons were excluded otherwise. Axonal arbors were analyzed by first counting the total number of branch points and collateral terminations in each cortical layer for each cell. This was done over the entire length of all axon collaterals originating from a single neuron until they entered either the marginal zone or white matter. In some cases, axons entering the white matter would later reenter the cortical layers. These axons were again followed and analyzed for branching and termination patterns.

The numbers of branch points and terminations were corrected for two factors that could inherently affect the probability of their occurrence, independent of the treatments. First, to compare arborization patterns in layer 5 with layers $2-4$, it was necessary to normalize for the difference in vertical widths (pial to white matter side) between these layers. For example, more branch points or terminations might be observed in layers 2-4 than layer 5 simply because of its greater width. Thus, cortical width was normalized between layer 5 and the wider cortical layers above it, layers $2-4$, by defining an equivalent distance (ed) just above layer 5 that we call layer 4 ed. This was done by counting only the branching and 
terminating events in layers $2-4$ that fell between the layer 5-4 border and the equivalent absolute width of layer 5 just above that border. The resultant numbers were compared between the two layers using a Wilcoxon signed rank test.

The second factor is inherent in the geometry of branching structures. A randomly branching tree will naturally have increased total numbers of branch points or terminal arbors farther from the base of the tree. Therefore, the numbers of branch points found in layer 5 and layer 4 ed were divided by the number of collateral axons that entered each cortical layer. These numbers reflect a branching rate per number of collaterals entering each cortical layer. The numbers of terminations were divided by the number of collateral axons entering the cortical layer plus the number of branch points within that layer. This reveals the probability that an axon collateral that either enters from outside the layer or originates from a branch within the layer will terminate in the layer. Because all collateral axons grew entirely in our culture system, this value includes only true terminations. In contrast, terminal arbors in acute slices $(\mathrm{Cal}-$ laway and Lieber, 1996) also represent axons that were cut during the slicing process. Therefore, a similar analysis of termination probabilities is less meaningful in acute slices.

To compare the arborization preference for layer 4 ed between the standard media and TTX groups, we calculated a preference value. The rate of branching or termination in layer 4 ed was divided by the sum of the rates in layer 4 ed and layer 5. A value of 0.5 represents no preference for either layer, whereas values $>0.5$ denote a preference to branch or terminate in layer 4 ed. The preference values between the two groups were statistically compared using a Mann-Whitney $U$ test.

\section{RESULTS}

Layer 6 pyramidal neurons developing in vivo arborize preferentially in layer 4 but form very few arbors in layer 5 (Callaway and Lieber, 1996). If intrinsic spontaneous activity or outside areas influence layer-specific connections, their removal could disrupt this specificity. Such disruptions could be dramatic, causing gross axonal targeting mistakes, or axonal targeting specificity could be simply reduced, resulting in increased "noisiness" in the layerspecific arborization pattern. Although not as striking as obvious targeting errors, such changes during development could have profound effects on cortical processing.

We therefore tested the possible roles for these factors in a system that allowed careful examination of axonal arborization patterns between cortical layers. Organotypic slice cultures were prepared from ferret area 17 before layer 6 pyramidal neurons develop ascending recurrent axon collaterals in overlying cortical layers (P14 and P15; Fig. 1), allowing us to examine their initial local axon development in the absence of extrinsic influences. At this stage, most layer $2 / 3$ neurons have completed their migration (McConnell, 1988; Jackson et al., 1989), and layer 5 can be clearly distinguished from layers 6 and 4 in Nissl-stained sections (Callaway and Lieber, 1996). Previous studies have shown that neuronal migration, layer formation, cellular morphology, and physiology develop normally in cortical explants (Caeser et al., 1989; Yamamoto et al., 1989, 1992; Bolz et al., 1990, 1992; Molnar and Blakemore, 1991; Götz and Bolz, 1992; Annis et al., 1993).

Slices in culture maintained clear laminar boundaries and normal cytoarchitecture as revealed from Nissl stain (Fig. 2A). The arrowhead in Figure $2 A$ indicates a single cell labeled in layer 6 , the bottom dark band. Above that, layer 5 is easily distinguishable as the lighter band of staining caused by large, sparsely spaced cell bodies. At this developmental stage the division between layers $2 / 3$ and 4 was not easily discernible; thus the zone above layer 5 containing both layers will be referred to as layers 2-4. All neurons included in this study were completely labeled with biocytin, allowing full visualization of axonal and dendritic processes (Fig. 2B). At higher power, en passant synaptic swellings
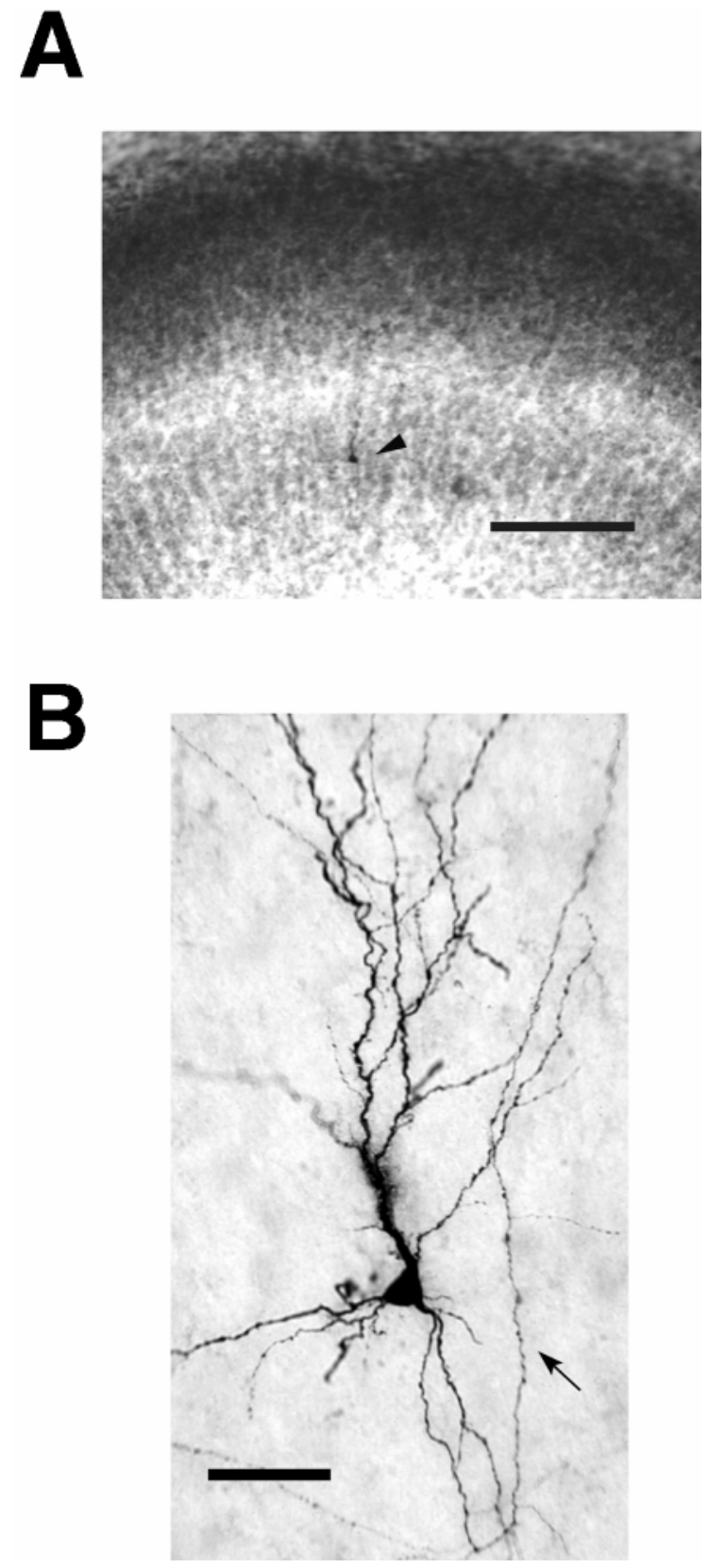

Figure 2. Photographs of an intracellularly labeled layer 6 pyramidal neuron in a ferret area 17 slice culture. $A$, Low-power view illustrating cortical layers revealed by staining with thionin. Layer 5 is distinct as the light band in the middle of the slice. The dark band below it is layer 6, containing a single labeled cell indicated by the arrowhead. Layers $2 / 3$ and 4 are not distinct at this stage in development and are referred together as layers 2-4. Slice is oriented with pial surface toward top. Scale bar, 200 $\mu \mathrm{m}$. $B$, High-power view showing quality of biocytin labeling and morphology of dendrites and axons that developed in culture. A recurrent axon collateral that originated from the white matter side of the cell body is denoted by the arrow. The small beads on the axon are synaptic swellings (Martin and Whitteridge, 1984), which are evenly spaced along the axon. Scale bar, $50 \mu \mathrm{m}$.

similar to those seen on axons labeled in vivo and in acute slices (Gilbert and Wiesel, 1979; Martin and Whitteridge, 1984; Buhl et al., 1994) could be seen evenly spaced along the axons in culture. Layer 6 pyramidal neurons developed in culture for 5-7 d, corresponding to an in vivo age of P19-P22 (P14 + 5 dic $-\mathrm{P} 15+7$ dic). 

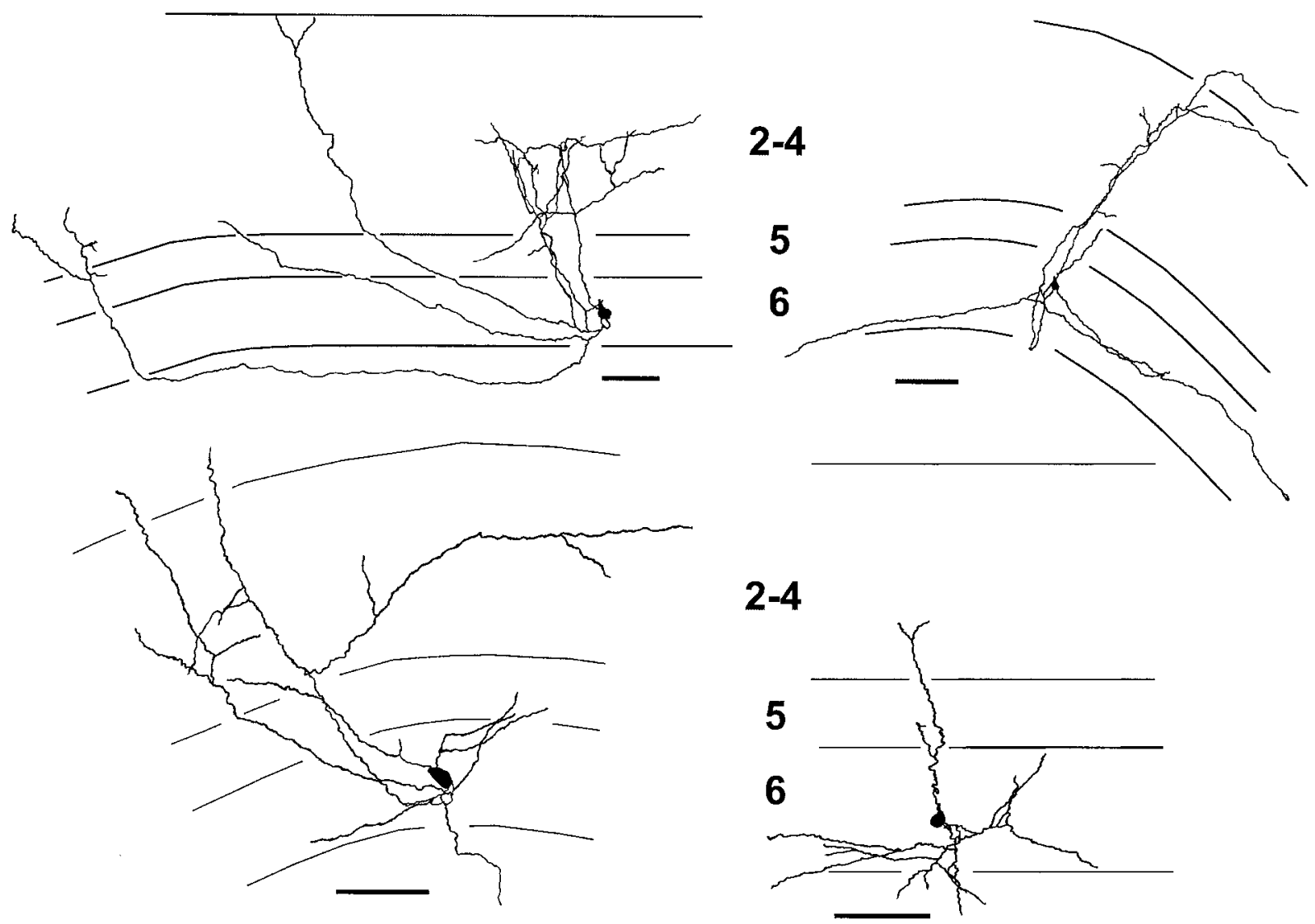

Figure 3. Camera lucida drawings illustrating typical axons from layer 6 pyramidal neurons grown in vitro. Dendrites are omitted for clarity. Most cells formed a sparse to moderate density of axonal arbors preferentially in layers 2-4, as exemplified by the cells on the top row and the bottom left quadrant. Usually, these axons were restricted to the region above the cell body, as seen in vivo. Sometimes, an axon collateral would project laterally up to 2 mm away from the cell body before arborizing in the cortical layers (cell in top left quadrant). The cell in the bottom right quadrant represents a less mature arbor pattern $(\mathrm{P} 14+5 \mathrm{dic})$ seen in five of the 24 cells in the sample. Axons are just beginning to ascend and branch in layer 4 . Such cells are very similar to in vivo cells labeled at P19-P20 (Fig. 1). Most cells appeared more mature, with arbor patterns and density similar to the remaining three cells. The solid polygons are cell bodies, and the fine horizontal lines indicate laminar borders. The most pial laminar border represents the beginning of layer 1 . Scale bars, $100 \mu \mathrm{m}$.

\section{Layer 6 pyramidal neurons arborize preferentially in layers 2-4 in vitro}

Examination of axonal arborization patterns formed in standard media in vitro revealed a remarkable similarity to layer 6 pyramidal neurons that developed in vivo (Callaway and Lieber, 1996). Camera lucida reconstructions of typical layer 6 pyramidal neurons from standard media cultures are shown in Figure 3. The general axonal behavior exhibited by neurons growing in culture was as follows. Collateral axons branched off the main descending axon in layer 6 and extended above the cell body through layer 5 , without forming many arbors, and then grew into target layers $2-4$, forming axonal branches and terminating growth. Five of the 24 cells in our sample appeared less mature than others. They had only one or two axon collaterals that grew above layer 6, entering layers 5 and 4. These axons either had not begun to arborize or formed a single branch specifically in layer 4 (Fig. 3, bottom right quadrant). This is similar to what is seen in acute slices aged P19-P20 (Fig. 1). The majority of the cells appeared more mature. These cells had 2-10 axon collaterals entering the overlying cortical layers with a sparse to moderate density of arbors mostly in layers 2-4. Because these axons grew entirely in culture, the full pattern obtained by these axons is represented for every cell. In other words, no arbors were cut during the slicing process.
To test quantitatively whether these axons preferentially arborized in layers $2-4$, the numbers of branch points and terminations in each layer for each cell grown in culture were scored (Table 1). There were $\sim 3.5$-fold more branches and almost 7 -fold

Table 1. Mean values for branch points and terminations per cell in all cortical layers in standard media and TTX

\begin{tabular}{llllll} 
& \multicolumn{2}{c}{ Standard media (24) } & & \multicolumn{2}{c}{ TTX (25) } \\
\cline { 2 - 3 } \cline { 5 - 6 } & Branch points & Terminations & & Branch points & Terminations \\
\hline Layer 6 & $6.2 \pm 0.6$ & $3.7 \pm 0.5$ & & $3.8 \pm 0.4$ & $1.3 \pm 0.3$ \\
Layer 5 & $1.1 \pm 0.4$ & $0.8 \pm 0.3$ & & $1.5 \pm 0.3$ & $0.8 \pm 0.2$ \\
Layer 4 ed & $2.2 \pm 0.5^{*}$ & $2.6 \pm 0.5^{* *}$ & & $1.4 \pm 0.4 \mathrm{~ns}$ & $1.5 \pm 0.5 \mathrm{~ns}$ \\
Layers 2-4 & $4.0 \pm 0.9$ & $5.4 \pm 1.0$ & & $3.1 \pm 0.9$ & $4.0 \pm 0.9$ \\
\hline
\end{tabular}

$\overline{\text { Mean }( \pm \text { SE) numbers of branch points and terminations per cell for all cells }(n)}$ labeled in cultures grown in standard media with or without $1 \mu \mathrm{m}$ TTX. Slices were cultured on P14-P15 and incubated for 5-7 d before cells were labeled. Values in layer 5 and layer 4 equivalent distance (ed) were compared for each cell, and the significance was evaluated for the population (Wilcoxon signed rank test). Asterisks represent values that were significantly higher in layer 4 ed vs. layer 5 levels. ns, No significant difference.

${ }^{*} p<0.05$.

${ }^{* *} p<0.01$. 
more terminations in layers 2-4 than found in layer 5. However, the apparent preference for arborization in layers 2-4 over layer 5 could be attributed to the fact that layers 2-4 are usually at least twice the width (from pial to white matter side) of layer 5, increasing the probability of branching or termination in those layers. To correct this, the numbers of branches and collateral terminations in all of layer 5 were compared with those in an equivalent distance in depth in layers $2-4$, beginning at the layer 5 border. For example, if the region of layer 5 above a particular layer 6 cell was $100 \mu \mathrm{m}$ wide, we counted only those axonal branches and terminations in layers $2-4$ that were within the deepest $100 \mu \mathrm{m}$. In addition to correcting for cortical thickness, this allowed us to focus on the region in which axons might first encounter their correct laminar target, layer 4.

Axons terminated more than three times as often in the equivalent distance of layer 4 (layer 4 ed) versus all of layer 5 (Table 1; $p<0.004$, Wilcoxon signed rank test, two-tailed; $n=24$ cells) and branched almost twice as often in layer 4 ed versus all of layer 5 (Table $1 ; p<0.02$ ).

\section{Differences in axonal growth between cells developing in vitro and in vivo did not affect the preference of axons for layer $\mathbf{4}$ ed}

There were two main differences in axonal behavior between cells developing in vitro and in vivo. For the same developmental period, axons developing in vitro had more branches and terminations in layers $2-4$ ( $p<0.01$, Mann-Whitney $U$ test; in vivo values from Callaway and Lieber 1996; P19-P20 group, data not shown). This was likely attributable to the fact that most P19-P20 layer 6 neurons in vivo had very few axons reaching layer 4 . There was no significant difference in the numbers of branches or terminations between the two conditions in layers 6 or $5(p>$ 0.1 ). Therefore, axons in culture were slightly accelerated in their growth into layer 4 but were not simply undergoing an overall increase in arborizations. Accelerated growth did not eliminate the specificity of arbors for layer 4 ed over layer 5 .

The second difference was that axons growing in culture sometimes extended long distances tangentially, up to $2 \mathrm{~mm}$ away from the cell body, before forming arbors in the cortical layers (Fig. 3, top left cell). These axons also appeared to be specific for layer 4 ed, despite their aberration from a normal growth trajectory directly above the cell body.

Finally, a single layer 6 pyramidal neuron labeled in culture clearly did not show a preference for layers 2-4 (Fig. 4) but arborized almost exclusively in layers 5 and 6 . This may not represent an "incorrect" projection, because a small population of layer 6 pyramidal neurons $(\sim 5 \%$ in the cat $)$ are known to project to the visual claustrum, not to the LGN, and their local axonal arbors prefer layers 5 and 6, not layer 4 (Katz, 1987). Claustrumprojecting layer 6 cells also usually have an apical dendrite extending into layer 1 , whereas apical dendrites of LGN-projecting layer 6 cells extend only as far as layer $2 / 3$. Unlike most other layer 6 pyramids in our sample, the one layer 6 neuron with axons preferring layers 5 and 6 also had an apical dendrite extending into layer 1 ( 2 of 24 cells had an apical dendrite in layer 1$)$. This observation suggests that this was a claustrum-projecting cell, making the correct local projections for its cell type. Because we did not definitively distinguish between different types of pyramidal neurons by retrograde labeling from subcortical target areas, all were included in the data set and our analyses (see above).

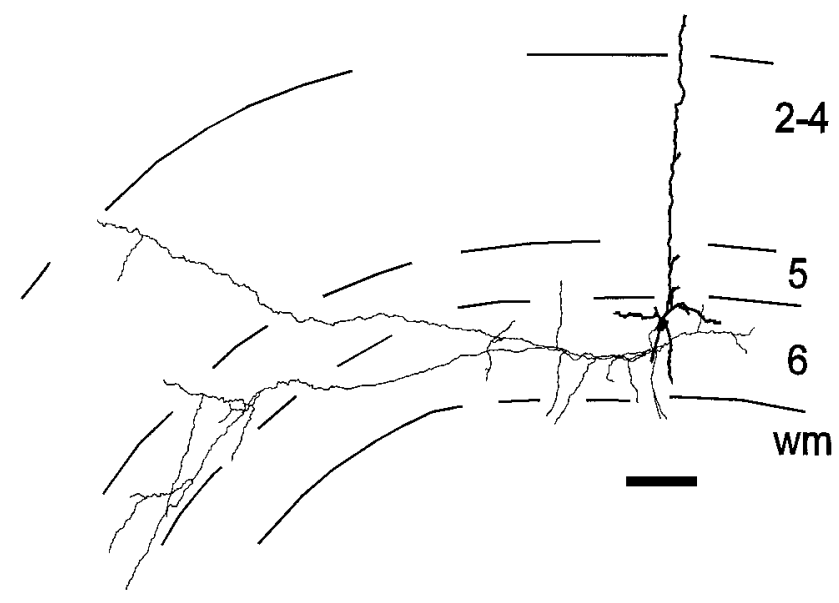

Figure 4. Presumptive "claustrum-projecting" layer 6 pyramidal neuron. In cat area 17, such cells have apical dendrites extending into layer 1 and local axonal arbors predominantly in layers 5 and 6 (Katz, 1987). This cell was unique in its preference to arborize in layer 5. Finer neuronal processes indicate axons and thicker ones indicate dendrites. Scale bar, $100 \mu \mathrm{m}$.

\section{Blockade of spontaneous activity in cortical slices reduced specificity of arborization}

The above results suggest that patterned spontaneous activity arising from extrinsic connections does not instruct layer 6 pyramidal neurons to arborize specifically in layer 4 . However, recent evidence has shown that spontaneous activity intrinsic to the cortex is important in the development of thalamocortical and local horizontal connections in the cortex (Herrmann and Shatz, 1995; Ruthazer and Stryker, 1996). Although it is unclear if the source of cortical spontaneous activity in vivo is autonomously generated or because of thalamic input (Mooney et al., 1996), cortical neuron cultures and explants have been reported to develop robust and somewhat synchronous spontaneous activity (Habets et al., 1987; Gutnick et al., 1989; Muramoto et al., 1993). To investigate the possible role of intrinsic spontaneous activity in the development of local, layer-specific connections, we added $1 \mu \mathrm{M}$ TTX to the media of approximately half of the cultures. Therefore, sodium-dependent action potentials were blocked in these cultures during the entire 5-7 $\mathrm{d}$ incubation period. This blockade was verified when slices were removed from culture for intracellular biocytin labeling of individual neurons (see Materials and Methods).

Camera lucida reconstructions representative of the range of morphologies in our sample of 25 TTX-treated cells are shown in Figure 5. These neurons appear to be forming just as many branches in layer 5 as in layer 4 ed. This apparent lack of specificity is verified by quantitative analyses (Table 1). There is not a statistically significant difference in the number of branches or terminations between layer 5 and layer 4 ed $(p>0.1$, Wilcoxon signed rank test). Nevertheless, there are still almost twice as many terminations in layer 4 ed as in layer 5 .

Comparisons between TTX and standard media neurons in Table 1 show a significant decrease in the numbers of branches and terminations in layer 6 in the TTX group ( $p<0.01$, comparisons of other layers are made in the next section). However, TTX did not decrease the number of collateral axons that entered layer 5 (mean \pm SEM in standard media, $3.13 \pm 0.34$; in TTX, $3.16 \pm 0.35 ; p>0.9$, Student's $t$ test) or layer 4 (mean \pm SEM in standard media, $3.17 \pm 0.46$; in TTX, $3.68 \pm 0.43 ; p>0.4$ ) between the two groups, suggesting that many of the axon col- 

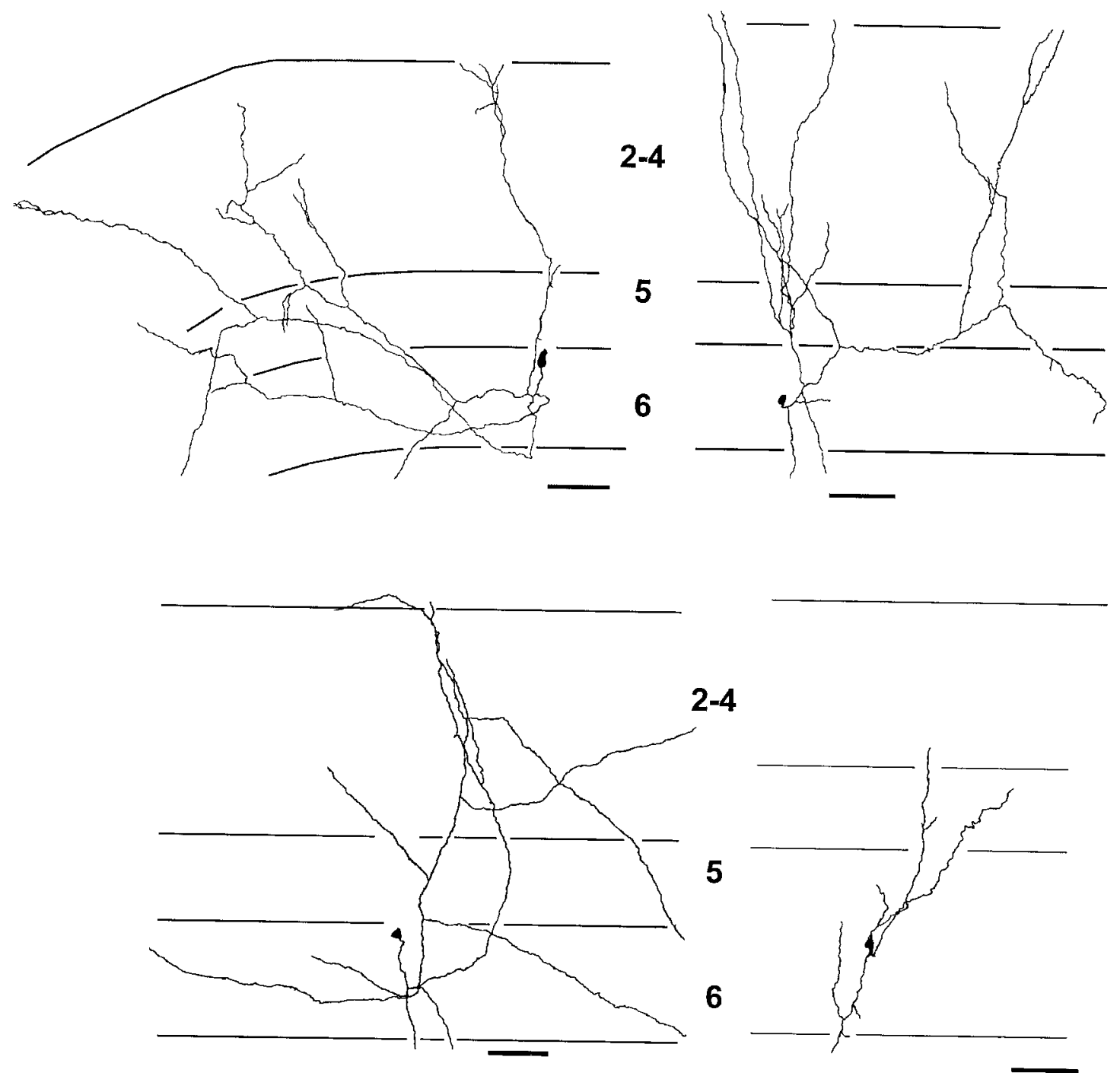

Figure 5. Camera lucida reconstructions of axonal arbors of four representative layer 6 pyramidal neurons cultured in media containing $1 \mu \mathrm{m}$ TTX. Unlike in standard media cultures, TTX-treated layer 6 pyramids formed as many or more axonal arbors in layer 5 than layers $2-4$. This is particularly noticeable for the cells in the top row, which have many axonal branches in layer 5 (7 dic). Conventions as in Figure 3 . Scale bars, $100 \mu \mathrm{m}$.

laterals that formed in layer 6 in standard media did not extend into layer 5. Both groups also had comparable numbers of axons that grew up to layer 1, suggesting there was not a difference in the acceleration of axon extension between the two treatments.

\section{TTX-treated cells fit a random branching model, but axons are still terminating preferentially in layer 4 ed}

The mean values in Table 1 suggest that TTX reduced the specificity for layer 4 ed of both axonal branches and terminations. However, it is unclear exactly how much of the effect is from a reduction of arbors in layer 4 ed, an increase in layer 5 , or an effect on just branches versus terminations. We wanted to determine the effect TTX was having on branching and terminating in layers 5 and 4 and to directly compare the relationship of these parameters in both layers between neurons grown in standard media or TTX.

To differentiate between possibilities, branching values were normalized to the number of axon collaterals entering each layer. This was important because the null hypothesis for specific arbor patterns is that axons branch randomly throughout the cortical layers. However, an inherent property of a randomly branching structure, such as a tree, is a larger number of branches farther away from the base of the tree. Each branching event lower on the tree gives rise to a new collateral, increasing the potential for branching higher on the tree. Therefore, to more carefully examine if these axons were displaying random branching patterns, the numbers of branch points in each layer were divided by the numbers of collateral axons that entered that layer. This resulted in a branch rate (branch points per axon collateral) in each cortical layer and normalized for the number of collateral axons that had the opportunity to branch in each layer.

The potential for axon termination is dependent not only on the number of axon collaterals that enter a cortical layer but also the number of branch points within that layer. Therefore, the number of terminations in each layer was divided by the sum of both values. This resulted in a termination probability, with a value of unity indicating that every axon collateral that entered or originated in that layer also terminated in that layer and a value of zero indicating that no such collaterals terminated in that layer. 
A.

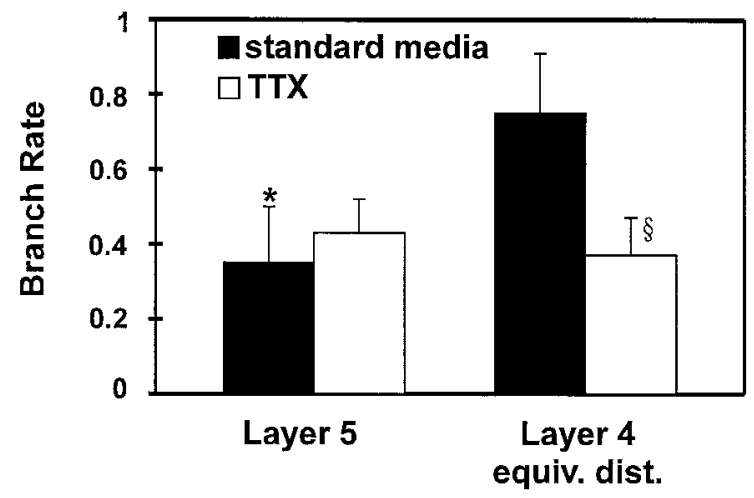

B.

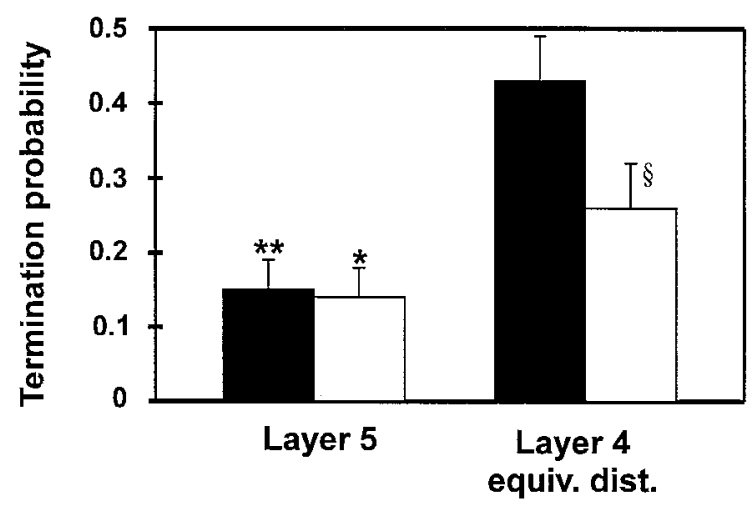

Figure 6. Mean branch rates and termination probabilities in layer 5 and layer 4 ed for cells from standard media (filled bars) and TTX groups (open bars). $A$, In standard media, the rate at which axon collaterals branch in layer 4 is significantly higher than layer 5 , but there is not a difference in TTX. $B$, For both standard media and TTX groups, the termination probability is significantly higher in layer 4 ed than layer 5 . Comparisons of values in layer 4 ed between the standard media and TTX groups show a reduction of the branch rates and termination probabilities in layer 4 ed. ${ }^{s} p=0.06$. Significant difference between values in layer 4 ed and layer $5: * p<0.05 ; * p<0.01$.

In standard media, the corrected values revealed differences between layer 5 and layer 4 ed (Fig. 6) similar to those obtained with the raw numbers of branches and terminations shown in Table 1 . The branch rate in layer 4 ed was two times larger than that in layer 5 ( $p=0.03$, Wilcoxon signed rank test), and the probability for an axon to terminate in layer 4 ed was almost three times greater $(p=0.0003)$. This indicates that these axons are not branching or terminating at a random rate but are clearly showing a strong preference for layer $4 \mathrm{ed}$.

In contrast, the axons of layer 6 pyramidal neurons treated with TTX did not branch specifically in layer 4 ed $(p>0.25$, Wilcoxon signed rank test) but still terminated preferentially in layer 4 ed $(p=0.05$; Fig. 6). Furthermore, comparison between the standard media and TTX groups indicates that TTX did not affect the branching rate or termination probability in layer $5(p>0.1$, Mann-Whitney $U$ Test; Fig. 6). However, the branch rate in layer 4 ed was reduced by almost $50 \%(p=0.06)$, and the termination probability was reduced by $60 \%$ in the TTX group $(p=0.06)$.

A direct comparison of each layer between the groups shows a strong trend toward reduction in specificity, but it does not address the differences in the relationship between layers 4 and 5 for
A.

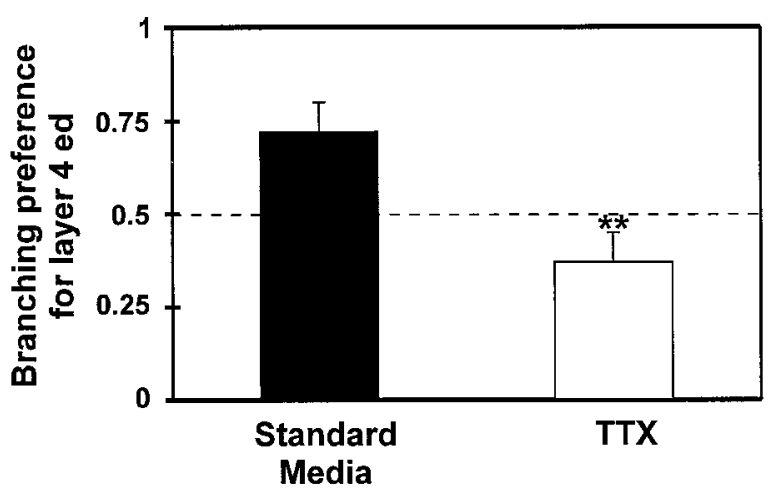

B.

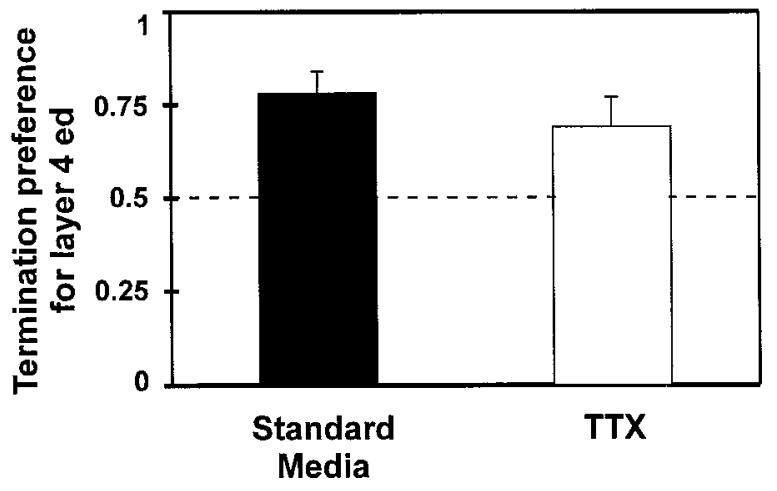

Figure 7. Comparison of arborization preference for layer 4 ed versus layer 5 between standard media and TTX cultures. Preference values were calculated (see Results) so that the relationship between layer 4 ed and layer 5 could be directly compared between the two groups. The dashed line corresponds to no preference for either layer. Values above the line correspond to a preference for layer 4 ed. $A$, Axons grown in standard media show a significantly greater preference to branch in layer 4 ed than those grown in TTX. $B$, However, the preference for axons to terminate in layer 4 ed was similar between the groups. $* * p<0.01$.

the two groups. To demonstrate differences in preference more clearly, preference values were calculated. These values are simply the proportions of branch rates or termination probabilities in layer 4 ed versus the totals in layer 4 ed and layer 5 (Fig. 7). A value of 0.5 corresponds to no preference for either layer, although values $>0.5$ correspond to a preference for layer 4 ed. This comparison shows that TTX resulted in a significant reduction of preferential branching in layer 4 ed versus layer 5 from a value of 0.72 to a value of 0.37 ( $p<0.005$, Mann-Whitney $U$ test; Fig. 7). But TTX treated axons displayed nearly as strong a preference to terminate in layer 4 ed as axons developing in standard media $(p>0.5)$.

Taken together, the above analyses reveal that layer 6 pyramidal neurons cultured in standard media do not branch or terminate at the same rate in all layers; rather, they do so preferentially in layer 4 ed versus layer 5. However, the blockade of spontaneous activity in organotypic slice cultures results in branching rates that are not significantly different between layer 4 ed and layer 5, with the greatest effect being a reduction in branching in layer 4 . TTX treatment does not significantly reduce the preference to terminate in layer 4 ed, although it does reduce the termination rate relative to that in standard media. 


\section{DISCUSSION}

Our findings are consistent with the hypothesis that layer-specific connections in the cortex develop using local molecular cues; however, they also implicate a role for cortical spontaneous activity. We found that layer 6 pyramidal neurons developing in organotypic slice culture preferentially arborized in layer 4 ed and avoided layer 5, as seen in vivo (Callaway and Lieber, 1996), even though no extrinsic influences were present. In contrast, blocking spontaneous activity in cortical slice cultures with TTX reduced this specificity such that branching in layers 5 and 4 was almost equivalent.

Specific arborization patterns achieved by layer 6 pyramidal neurons could arise from inhibition of branching in layer 5 , branch-promoting cues in layer 4 , or a combination of both. To distinguish between these possibilities, we normalized arbor values in each layer by the number of collaterals within that layer. This enabled us to discern the effects that TTX had on arborization parameters in each layer independent of confounding geometrical relationships inherent in branching axonal trees. The analysis revealed that in standard media, the rate at which axons branch in layer 4 is much greater than in layer 5. Previous studies have shown that disruption of expression of an inhibitory cue results in increased axonal branching in nontarget regions (Walter et al., 1987). Therefore, if blocking spontaneous activity neutralizes an inhibitory cue in layer 5, we would expect to see an increase in the branching rate after TTX application. However, no significant branching increase was seen in layer 5, suggesting that TTX does not affect putative inhibitory cues in layer 5 . Instead, we observed a selective decrease in the branch rate in layer 4, implicating a role for branch-promoting cues in layer 4.

Our activity blockade results are consistent with two scenarios: (1) neurons may require spontaneous activity to properly respond to available cues, suggesting there is a branch-promoting cue in layer 4 that these axons can no longer reliably detect in the presence of TTX; or (2) TTX might disrupt the proper production or secretion of a branch-promoting cue in layer 4. The implication of such a cue in layer 4 concurs with results from Castellani and Bolz (1997). Using a stripe assay of membrane carpets from different cortical layers of postnatal rat cortex (P9$\mathrm{P} 11$ ), ventricular zone explants (embryonic days 15 and 16) containing mostly layer 6 cells were given the choice to grow fibers on carpets from target or nontarget layers. Fibers grew and branched preferentially on carpets prepared from target layers for layer 6 neurons. Heat inactivation of carpets resulted in a significant decrease in branching in target layers and a slight decrease in layer 5. This further suggests that if layer 5 is at all inhibitory, its effect is much weaker than the branch-promoting environment in layer 4.

An alternative hypothesis to explain our results could be that TTX is not reducing specificity of arbors but merely reducing the total extent of arborizations by an overall decrease in growth of axons. However, there are two lines of evidence against this possibility: (1) there is not a decrease in the number of total collaterals or the length to which they grow in the TTX group; and (2) the decrease in arbors in the TTX group is specific to the two main target layers 6 and 4 and is not seen in layer 5 .

In accordance with the above results, the putative molecular cues that initiate branching of layer 6 axons should act in a layer-specific manner and show activity-dependent expression or responsiveness. Neurotrophins are qualified candidate molecules fulfilling these criteria. Increasing evidence suggests that neuro- trophins can act in a layer-specific manner. In ferret slice cultures, brain-derived neurotrophic factor selectively increases the complexity of basal dendritic arbors in layer 4 neurons, whereas NT-4 does so in layers 5 and 6 (McAllister et al., 1995). Furthermore, these effects are dependent on the presence of electrical activity, which appears to affect both proper expression and action of neurotrophic factors (McAllister et al., 1996). In addition, more recent experiments suggest neurotrophic factors may also affect layer-specific axonal branching and growth when applied exogenously to cortical explants (Bolz et al., 1997). For example, basic fibroblast growth factor (bFGF) and NT-3 selectively increase axonal arbors of layer 6 neurons but not of layer $2 / 3$ neurons in vitro. Based on these experiments and others implicating a role for neurotrophins in the elaboration of axonal arbors (CohenCory and Fraser, 1995) and our observation that axonal branching decreases in the absence of $\mathrm{Na}^{+}$-dependent activity, it is an intriguing possibility that interactions between activity and neurotrophins are important for the formation of layer-specific connections.

Activity is generally thought to sculpt the patterns of laterformed connections in the cortex, such as the segregation of LGN axons into ocular dominance columns (LeVay et al., 1978; Antonini and Stryker, 1993) or the clustering of horizontal connections (Callaway and Katz, 1991; Löwel and Singer, 1992; Ruthazer and Stryker, 1996). In contrast, earlier-formed layer-specific connections are thought not to require activity (Goodman and Shatz, 1993; Katz and Shatz, 1996). Our results suggest that spontaneous activity may, in fact, play an integral role in development of layer-specific circuitry by influencing the expression or detection of molecular cues.

Furthermore, this complicates a simple dichotomy of activitydependent versus activity-independent mechanisms. The action of electrical activity and molecular cues may be tightly interdependent (Rutherford et al., 1997). Recent evidence also suggests a role for activity in the development of earlier-formed thalamocortical connections. TTX infusion in the cortex of neonatal cats before thalamic afferents have grown into the subplate results in a drastic reduction of axonal branches in layer 4 and elongation of axons past the supragranular layers into the marginal zone (Herrmann and Shatz, 1995).

For proper axonal targeting, cues must instruct axons not only to form branches in the correct regions but also to appropriately terminate extension. Are the same cues doing both jobs? We found that blockade of spontaneous activity did not have the same effect on terminations as it did on branching. Axons grown in standard media had a higher probability of terminating in layer 4 over layer 5 . This preference for layer 4 was not significantly different between neurons grown in standard media and TTX, although there was still a fairly large decrease in the overall termination probability in layer 4 . This suggests that the regulation of mechanisms affecting branching and growth is not the same. Other studies also suggest a dissociation between mechanisms affecting these parameters. The neurotrophin bFGF selectively increases branching of axons without affecting growth rate in dissociated cultures of hippocampal neurons (Aoyagi et al., 1994). Also, bFGF can selectively influence axonal branching behavior of layer 6 cortical neurons in vitro (Bolz and Castellani, 1997).

A level of complexity that can be added to a simple model of layer-specific cue expression is the existence of a heterogeneous group of cells within a layer that have different local and efferent axonal targets. In cat area 17, layer 6 contains at least two types 
of pyramidal projection neurons. The majority project locally to layer 4 and extrinsically to the LGN. A smaller population $(\sim 5 \%)$ projects locally to layers 5 and 6 and has efferent connections to the visual claustrum (Katz, 1987). This second class of neurons has not been investigated in ferret, but its close relative the mink has connections from area 17 to the visual claustrum, although the layer of origin has not been demonstrated (McConnell and LeVay, 1986). One of the 24 pyramidal neurons that we labeled from standard media cultures had the typical characteristics of a layer 5, claustrum-projecting cell. Therefore, different cell types might respond differentially to molecular cues available in the cortical slice. This suggests the presence of different expression of cues and/or receptors for cue detection or the same cues coupled to different accessory molecules or intracellular pathways.

In conclusion, layer 6 pyramidal neurons specifically arborize in layer 4 in the absence of extrinsic influences that could arise from connections with outside areas. However, intrinsic cortical activity is required in the slice culture for axons to form arbors preferentially in layer 4 . In the presence of TTX, branching rates are dramatically reduced in layer 4 but are not significantly affected in layer 5. Thus, an interplay between molecular cues and spontaneous activity may prove important in sculpting the precise layer-specific connections of the mammalian cerebral cortex.

\section{REFERENCES}

Annis CM, Robertson RT, O’Dowd DK (1993) Aspects of early postnatal development of cortical neurons that proceed independently of normally present extrinsic influences. J Neurobiol 24:1460-1480.

Antonini A, Stryker MP (1993) Development of individual geniculocortical arbors in cat striate cortex and effects of binocular impulse blockade. J Neurosci 13:3549-3573.

Aoyagi A, Nishikawa K, Saito H, Abe K (1994) Characterization of basic fibroblast growth factor-mediated acceleration of axonal branching in cultured rat hippocampal neurons. Brain Res 661:117-126.

Blanton MG, Lo Turco JJ, Kriegstein AR (1989) Whole cell recording from neurons in slices of reptilian and mammalian cerebral cortex. J Neurosci Methods 30:203-210.

Blochl A, Thoenen H (1995) Characterization of nerve growth factor (NGF) release from hippocampal neurons: evidence for a constitutive and an unconventional sodium-dependent regulated pathway. Eur J Neurosci 7:1220-1228.

Bolz J, Castellani V (1997) How do wiring molecules specify cortical connections? Cell Tissue Res 290:307-314.

Bolz J, Novak N, Götz M, Bonhoeffer T (1990) Formation of targetspecific neuronal projections in organotypic slice cultures from rat visual cortex. Nature 346:359-362.

Bolz J, Novak N, Staiger V (1992) Formation of specific afferent connections in organotypic slice cultures from rat visual cortex cocultured with lateral geniculate nucleus. J Neurosci 12:3054-3070.

Bolz J, Castellani V, Batardière A (1997) Neurotrophic factors play a role in the elaboration of local cortical circuits. Soc Neurosci Abstr 23:566.

Bonhoeffer T (1996) Neurotrophins and activity dependent development of the neocortex. Curr Opin Neurobiol 6:119-126.

Buhl EH, Halasy K, Somogyi P (1994) Diverse sources of hippocampal unitary inhibitory postsynaptic potentials and the number of synaptic release sites. Nature 368:823-828.

Caeser M, Bonhoeffer T, Bolz J (1989) Cellular organization and development of slice cultures from rat visual cortex. Exp Brain Res 77:234-244.

Callaway EM (1998) Prenatal development of layer-specific local circuits in primary visual cortex of the macaque monkey. J Neurosci 18:1505-1527.

Callaway EM, Katz LC (1991) Effects of binocular deprivation on the development of clustered horizontal connections in cat striate cortex. Proc Natl Acad Sci USA 88:745-749.

Callaway EM, Katz LC (1992) Development of axonal arbors of layer 4 spiny neurons in cat striate cortex. J Neurosci 12:570-582.

Callaway EM, Lieber JL (1996) Development of axonal arbors of layer 6 pyramidal neurons in ferret primary visual cortex. J Comp Neurol 376:295-305.

Callaway EM, Wiser AK (1996) Contributions of individual layer 2/5 spiny neurons to local circuits in macaque primary visual cortex. Vis Neurosci 13:907-922.

Castellani V, Bolz J (1997) Membrane-associated molecules regulate the formation of layer-specific cortical circuits. Proc Natl Acad Sci USA 94:7030-7035.

Changeux JP, Danchin A (1976) Selective stabilization of developing synapses as a mechanism for the specification of neuronal networks. Nature 264:705-712.

Cohen-Cory S, Fraser SE (1995) Effects of brain-derived neurotrophic factor on optic axon branching and remodelling in vivo. Nature 378:192-196.

Fryer RH, Kaplan DR, Feinstein SC, Radeke MJ, Grayson DR, Kromer LF (1996) Developmental and mature expression of full-length and truncated TrkB receptors in the rat forebrain. J Comp Neurol 374:21-40.

Gilbert CD (1983) Microcircuitry of the visual cortex. Annu Rev Neurosci 6:217-247.

Gilbert CD, Wiesel TN (1979) Morphology and intracortical projections of functionally characterized neurones in the cat visual cortex. Nature 280:120-125.

Goodman CS, Shatz CJ (1993) Developmental mechanisms that generate precise patterns of neuronal connectivity. Cell 72:77-98.

Goodman LJ, Valverde J, Lim F, Geschwind MD, Federoff HJ, Geller AI, Hefti F (1996) Regulated release and polarized localization of brain-derived neurotrophic factor in hippocampal neurons. Mol Cell Neurosci 7:222-238.

Götz M, Bolz J (1992) Formation and preservation of cortical layers in slice cultures. J Neurobiol 23:783-802.

Gutnick MJ, Wolfson B, Baldino Jr F (1989) Synchronized neuronal activities in neocortical explant cultures. Exp Brain Res 76:131-140.

Habecker BA, Tresser SJ, Rao MS, Landis SC (1995) Production of sweat gland cholinergic differentiation factor depends on innervation. Dev Biol 167:307-316.

Habets AM, Van Dongen AM, Van Huizen F, Corner MA (1987) Spontaneous neuronal firing patterns in fetal rat cortical networks during development in vitro: a quantitative analysis. Exp Brain Res 69:43-52.

Herrmann K, Shatz CJ (1995) Blockade of action potential activity alters initial arborization of thalamic axons within cortical layer 4. Proc Natl Acad Sci USA 92:11244-11248.

Jackson CE, Peduzzi JD, Hickey TL (1989) Visual cortex development in the ferret. I. Genesis and migration of visual cortical neurons. J Neurosci 9:1242-1253.

Katz LC (1991) Specificity in the development of vertical connections in cat striate cortex. Eur J Neurosci 3:1-9.

Katz LC (1987) Local circuitry of identified projection neurons in cat visual cortex brain slices. J Neurosci 7:1223-1249.

Katz LC, Callaway EM (1992) Development of local circuits in mammalian visual cortex. Annu Rev Neurosci 15:31-56.

Katz LC, Shatz CJ (1996) Synaptic activity and the construction of cortical circuits. Science 274:1133-1138.

Kawamoto Y, Nakamura S, Nakano S, Oka N, Akiguchi I, Kimura J (1996) Immunohistochemical localization of brain-derived neurotrophic factor in adult rat brain. Neuroscience 74:1209-1226.

LeVay S, Stryker MP, Shatz CJ (1978) Ocular dominance columns and their development in layer IV of the cat's visual cortex: a quantitative study. J Comp Neurol 179:223-244.

Löwel S, Singer W (1992) Selection of intrinsic horizontal connections in the visual cortex by correlated activity. Science 255:209-212.

Lund JS (1988) Anatomical organization of macaque monkey striate visual cortex. Annu Rev Neurosci 11:253-288.

Lund JS, Boothe RG, Lund RD (1977) Development of neurons in the visual cortex (area 17) of the monkey (Macaca nemestrina): a Golgi study from fetal day 127 to postnatal maturity. J Comp Neurol 176:149-188.

Martin KA, Whitteridge D (1984) Form, function, and intracortical projections of spiny neurones in the striate visual cortex of the cat. J Physiol (Lond) 353:463-504.

McAllister AK, Lo DC, Katz LC (1995) Neurotrophins regulate dendritic growth in developing visual cortex. Neuron 15:791-803.

McAllister AK, Katz LC, Lo DC (1996) Neurotrophin regulation of cortical dendritic growth requires activity. Neuron 17:1057-1064. 
McConnell SK (1988) Fates of visual cortical neurons in the ferret after isochronic and heterochronic transplantation. J Neurosci 8:945-974.

McConnell SK, LeVay S (1986) Anatomical organization of the visual system of the mink, Mustela vison. J Comp Neurol 250:109-132.

Molnar Z, Blakemore C (1991) Lack of regional specificity for connections formed between thalamus and cortex in coculture. Nature 351:475-477.

Mooney R, Penn AA, Gallego R, Shatz CJ (1996) Thalamic relay of spontaneous retinal activity prior to vision. Neuron 17:863-874.

Mozer BA, Benzer S (1994) Ingrowth by photoreceptor axons induces transcription of a retrotransposon in the developing Drosophila brain. Development 120:1049-1058.

Muramoto K, Ichikawa M, Kawahara M, Kobayashi K, Kuroda Y (1993) Frequency of synchronous oscillations of neuronal activity increases during development and is correlated to the number of synapses in cultured cortical neuron networks. Neurosci Lett 163:163-165.

O'Leary DD, Koester SE (1993) Development of projection neuron types, axon pathways, and patterned connections of the mammalian cortex. Neuron 10:991-1006.

Ruthazer E, Stryker M (1996) The role of activity in the development of long-range horizontal connections in area 17 of the ferret. J Neurosci 16:7253-7269.

Rutherford LC, DeWan A, Lauer HM, Turrigiano GG (1997) Brainderived neurotrophic factor mediates the activity-dependent regulation of inhibition in neocortical cultures. J Neurosci 17:4527-4535.
Stevens LM, Landis SC (1988) Developmental interactions between sweat glands and the sympathetic neurons which innervate them: effects of delayed innervation on neurotransmitter plasticity and gland maturation. Dev Biol 130:703-720.

Thoenen H (1995) Neurotrophins and neuronal plasticity. Science 270:593-598.

Walter J, Henke-Fahle S, Bonhoeffer F (1987) Avoidance of posterior tectal membranes by temporal retinal axons. Development 101:909-913.

Williams CV, Nordquist D, McLoon SC (1994) Correlation of nitric oxide synthase expression with changing patterns of axonal projections in the developing visual system. J Neurosci 14:1746-1755.

Wu W, Gloster A, Miller FD (1997) Transcriptional repression of the growth-associated $\mathrm{T}$ alpha1 alpha-tubulin gene by target contact. J Neurosci Res 48:477-487.

Xie K, Wang T, Olafsson P, Mizuno K, Lu B (1997) Activity-dependent expression of NT-3 in muscle cells in culture: implications in the development of neuromuscular junctions. J Neurosci 17:2947-2958.

Yamamoto N, Kurotani T, Toyama K (1989) Neural connections between the lateral geniculate nucleus and visual cortex in vitro. Science 245:192-194.

Yamamoto N, Yamada K, Kurotani T, Toyama K (1992) Laminar specificity of extrinsic cortical connections studied in coculture preparations. Neuron 9:217-228. 